\title{
SANS Study of Poly(ethylene glycol) Solutions in $\mathrm{D}_{2} \mathrm{O}$
}

\author{
G. Lancz ${ }^{a, *}$, M.V. Avdeev ${ }^{b}$, V.I. Petrenko ${ }^{b, c}$, V.M. Garamus ${ }^{d}$, M. Konerackáa $^{a}$ \\ AND P. KOPČANSKÝ ${ }^{a}$ \\ ${ }^{a}$ Institute of Experimental Physics, Slovak Academy of Sciences, Watsonova 47, 04001 Košice, Slovakia \\ ${ }^{b}$ Frank Laboratory of Neutron Physics, Joint Institute for Nuclear Research \\ Joliot-Curie 6, 141980 Dubna, Moscow region, Russia \\ ${ }^{c}$ Physics Department, Taras Shevchenko Kyiv National University, Volodymyrska 64, 01601 Kyiv, Ukraine \\ ${ }^{d}$ GKSS Research Centre, Max-Planck-Str. 1, 21502 Geesthacht, Germany
}

\begin{abstract}
Poly(ethylene glycol) is used for coating of colloidal particles and other surfaces for gaining biocompatibility. Particularly, it can be introduced into magnetic fluids. The aim of the current study was to reveal the structural characteristics of poly(ethylene glycol) in neat $\mathrm{D}_{2} \mathrm{O}$ using small-angle neutron scattering technique. Solutions of poly (ethylene glycol) (at temperature of $37^{\circ} \mathrm{C}$ ) with different molecular weights in an interval of 400-20000 were investigated. It is concluded that at low concentrations (less than $2 \%$ of mass fraction) poly(ethylene glycol) molecules behave as Gaussian coils.
\end{abstract}

PACS numbers: 36.20.-r, 61.05.fg

\section{Introduction}

Hydrophilic poly(ethylene glycol) (PEG) is used for coating of colloidal particles for gaining biocompatibility. Its introduction into magnetic fluids (liquid dispersions of magnetic nanoparticles) may increase the circulation time of magnetic particles in organisms by hindering the action of the mononuclear phagocyte system (reticuloendothelial system), which is a part of the immune system. This is because nanoparticles, or surfaces in general, coated with PEG show enhanced resistance against protein adsorption, see e.g. [1] and references therein. An increase in biocompatibility of a given magnetic fluid is expected even by the simple addition of PEG, i.e. without a covalent bond between PEG and the magnetic particle.

The goal of this study was to understand the structural characteristics of PEG molecules in water, which give some basic knowledge useful for further investigations of complex systems comprising PEG.

In the given work the solutions of PEG in deuterated water $\left(\mathrm{D}_{2} \mathrm{O}\right)$ with different molecular weights in a range of $M_{n}=400-20000$ were investigated using small-angle neutron scattering (SANS) technique. Recently [2, 3], SANS was successfully applied for PEG in $\mathrm{D}_{2} \mathrm{O}$ within a narrow range of $M_{n}=2000-8000$ at different solution ion strength. Together with the compressibility and intermolecular distance as a function of PEG concentration [2], two possible structures (Gaussian coils and flat

* corresponding author; e-mail: glancz@saske.sk "plates") of PEG were discussed [3] with respect to the scaling in the scattering as a function of $M_{n}$. Here, SANS characterization of PEG covers wider $M_{n}$-interval. Additionally, the measurements were performed at the physiological temperature of $37^{\circ} \mathrm{C}$ taking into account a specific interest for using PEG in biocompatible ferrofluids.

\section{Materials and methods}

PEG with four different molar masses was purchased from Sigma-Aldrich ("average mol wt. 400", "typical $M_{n}$ 1000", and "16000-24000") and Merck ("9000-11250 g/mol"). PEG with $M_{n}=400,1000$, 10000,20000 was dissolved in pure $\mathrm{D}_{2} \mathrm{O}$ (D-content $99.9 \%$ with the mass fractions within an interval of $0.5-10 \% . \quad \mathrm{D}_{2} \mathrm{O}$ was used to achieve a sufficient scattering contrast between PEG and the liquid carrier, as well as for reduction of the incoherent scattering background from hydrogen. SANS experiments were performed using the SANS-1 instrument located at the Neutron Facility at GKSS Research Centre, Geesthacht, Germany. Measurements were done at the temperature of $37^{\circ} \mathrm{C}$. For solutions of PEG with $M_{n}=400$, the $5 \mathrm{~mm}$ thick quartz cells were used. In other cases the thickness of the cells was $2 \mathrm{~mm}$. In all cases pure $\mathrm{D}_{2} \mathrm{O}$ was used as a buffer (blank, background sample). To obtain the differential cross-section per sample volume (hereafter referred to as scattered intensity) in the absolute scale $\left(\mathrm{cm}^{-1}\right)$ the standard calibration [4] using the scattering from $1 \mathrm{~mm}$ water sample was made after the background, buffer $\left(\mathrm{D}_{2} \mathrm{O}\right)$ and empty cell corrections. 


\section{Results and discussion}

Changes in the experimental SANS intensities are followed in Fig. 1 (examples are given for PEG 1000 and PEG 10000). One can see that at the PEG concentrations of less than $3 \%$ the scattering shows a pronounced Guinier-type behaviour corresponding to a form factor of the polymer coils. At higher concentrations the correlations between coils results in the structure-factor effect at small $q$-values, which is rather different for masses below 1000 and above 10000. Our interest in this study was the analysis of the coil form factor. For this purpose the scattering data from low-concentrated $(<3 \%)$ solutions were first approached by the Guinier law to reveal the radius of gyration of the coil, $R_{\mathrm{g}}$, at sufficiently small $q$-values $\left(q R_{\mathrm{g}} \leq 1\right)$. For low $M_{n}(400$ and 1000) the Guinier approximation is valid over the whole $q$-range covered in the experiments. This made possible to fit additionally the residual incoherent background caused by non-compensated hydrogen in PEG. It is important that this background was consistent for solutions with the same PEG concentrations independently of $M_{n}$.

The Guinier approximations for 1\% solutions of PEG with different masses are shown in Fig. 2. The dependence of the obtained radius of gyration on the PEG molecular mass in this case (inset to Fig. 2) reveals a power-law behaviour (line in the double logarithmic scale) with an exponent of $0.48 \pm 0.02$. This corresponds well to Gaussian coils (exponent $1 / 2$ ) and coincides with the result for PEG with $M_{n}=2000-8000$ in ionic solutions [3].

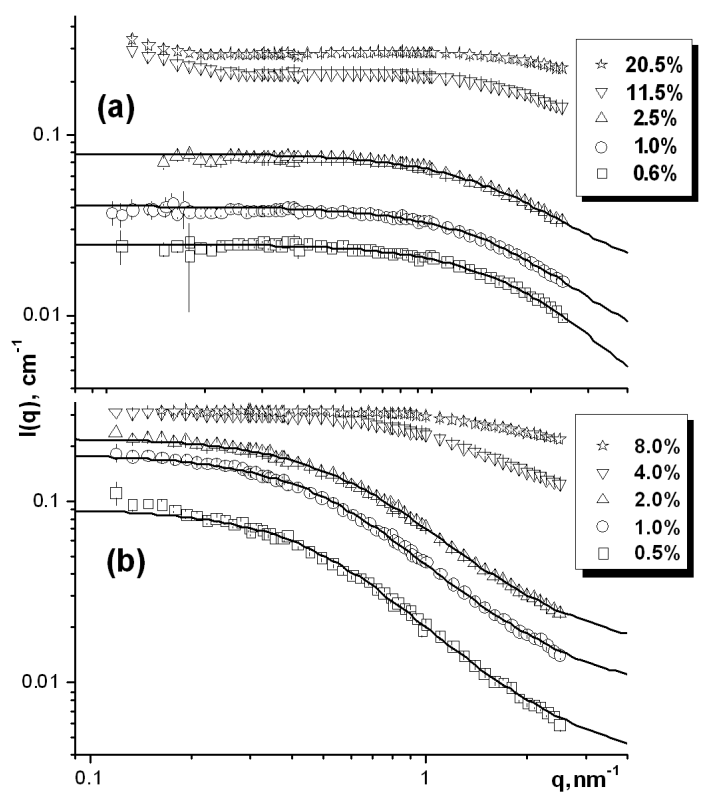

Fig. 1. Changes in SANS intensity (dots) with variation in mass fraction of PEG dissolved in $\mathrm{D}_{2} \mathrm{O}, M_{n}$ is 1000 (a) and 10000 (b). Solid lines show fits in accordance with the Debye formula (1) for non-interacting Gaussian coils.

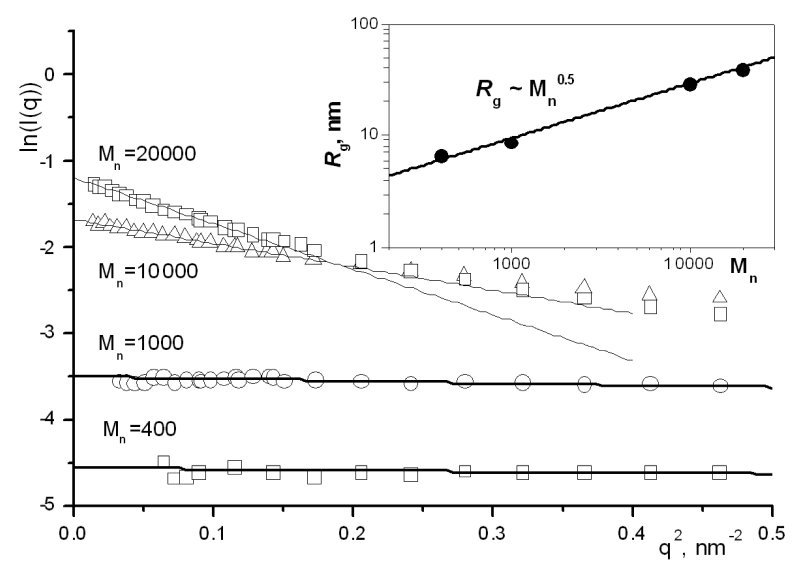

Fig. 2. Guinier plots of SANS intensity (dots) for $1 \%$ PEG solutions in $\mathrm{D}_{2} \mathrm{O}$ with different $M_{n}$ and corresponding linear approximations (lines) to regions $q R_{\mathrm{g}}<1$. Non-compensated incoherent background is subtracted. Several points with very low statistics in beginning parts of plots for $M_{n}=400$ and 1000 are removed from treatment. Inset shows dependence of obtained radius of gyration on polymer molecular weight. Experimental errors do not exceed the size of dots.

Hence, the Debye formula for the scattering from non-interacting Gaussian coils can be used at low PEG concentrations for any $M_{n}$. This is demonstrated in Fig. 1, where for diluted PEG solutions the fits are based on the formula

$$
I(q)=2 I_{0}\left[\mathrm{e}^{-x}-(1-x)\right] / x^{2}+B, \quad x=\left(q R_{\mathrm{g}}\right)^{2} .
$$

Here, $I_{0}=I(q \rightarrow 0)$ is the forward scattering intensity and $B$ is the residual background. The same fit quality takes place for similar solutions with $M_{n}$ of 400 and 20000. It should be noted that the relative difference in the values of the radius of gyration from the fit of the Debye formula (Eq. (1)) and from the Guinier approximation is below $5 \%$, and the Guinier approximation almost coincides with $(1)$ in the overlapping region $\left(q R_{\mathrm{g}}<1\right)$. Additionally, dependence of $q^{2} I(q)$ vs. $q$ reveals a typical behaviour for Gaussian polymer chains (not shown).

\section{Conclusion}

The observed scaling law $R_{\mathrm{g}} \sim M_{n}^{0.5}$ in a wide polymer mass interval (400-20000) strongly confirms the Gaussian coil structure of PEG in water solutions. The scattering form factor of the coils is well described by the Debye formula over the whole measured $q$-interval at the PEG concentration below $2 \%$. High adsorption properties of PEG for various substrates [5-7] and nanoparticles [8] also suggest a developed structure of this polymer.

\section{Acknowledgments}

This research project has been supported by the European Commission under the 7th Framework Programme 
through the "Research Infrastructures" action of the "Capacities" Programme, contract No. CP-CSA_INFRA-2008-1.1.1 number 226507-NMI3. It was also supported by the projects Nos. 26220220005 and 26220120033 in the frame of the Structural Funds of European Union. Projects VEGA 0077 and APVV-99-026505 are acknowledged, too.

\section{References}

[1] Y. Zhang, N. Kohler, M. Zhang, Biomaterials 23, 1553 (2002).

[2] K.A. Rubinson, J. Hubbard, Polymer 50, 2618 (2009).

[3] K.A. Rubinson, S. Krueger, Polymer 50, 4852 (2009).
[4] G.D. Wignall, F.S. Bates, J. Appl. Crystallogr. 20, 28 (1987).

[5] E. Tronel-Peyroz, H. Raous, D. Schuhmann, J. Coll. Interface Sci. 92, 136 (1983).

[6] A.M. Mota, M.L. Simões Gonçalves, J.P. Farinha, J. Buffle, Coll. Surf. A 90, 271 (1994).

[7] J.C. Dijt, M.A. Cohen Stuart, J.E. Hofman, G.J. Fleer, Coll. Surf. 51, 141 (1990).

[8] M.V. Avdeev, A.V. Feoktystov, P. Kopčanský, G. Lancz, V.M. Garamus, R. Willumeit, M. Timko, M. Koneracká, V. Závišová, N. Tomašovičová, A. Juríková, K. Csach, L.A. Bulavin, J. Appl. Crystallogr. 43, 959 (2010). 